\title{
Hydrogels Loaded with Methylene Blue: Sorption-Desorption and Antimicrobial Photoactivation Study
}

\author{
O. Nadtoka $(\mathbb{D}$, P. Virych $(\mathbb{D}$, and N. Kutsevol $\mathbb{D}$ \\ Chemical Department, Taras Shevchenko National University of Kyiv, City of Kyiv 01601, Ukraine \\ Correspondence should be addressed to P. Virych; sphaenodon@ukr.net
}

Received 7 February 2020; Accepted 9 April 2020; Published 27 April 2020

Guest Editor: Juan Li

Copyright (C) 2020 O. Nadtoka et al. This is an open access article distributed under the Creative Commons Attribution License, which permits unrestricted use, distribution, and reproduction in any medium, provided the original work is properly cited.

\begin{abstract}
Cross-linked dextran-graft-polyacrylamide hydrogels with different cross-linking density loaded with methylene blue was prepared as light-activated antimicrobial materials. Sorption/desorption properties of hydrogels were studied by estimating the change in the absorption maximum of MB solutions. The sorption/desorption rate of MB into/out of hydrogel depends on the hydrogel structure. The antibacterial properties of the light-irradiated materials were tested against Staphylococcus aureus. It was shown that irradiation of hydrogels in the presence of methylene blue was able to eliminate the bacteria strains.
\end{abstract}

\section{Introduction}

Bacterial infection usually occurs in burns, wounds, and lots of surgical operations [1]. Microorganisms play an essential role in the development and maintenance of pathologies, and their removal during the wound healing or biomechanical preparation is important to the success of treatment. To eliminate or reduce bacterial colonization, methods that included the use of high-dose antibiotic were used. The antibiotic is largely successful but can lead to the development of antibiotic-resistant strains of bacteria. One novel approach for the elimination of bacteria on different surfaces is to use a light-activated antimicrobial agent (LAAA) $[2,3]$.

Light-activated antimicrobial agents when excited with light of an appropriate wavelength, namely, with red light, generate singlet oxygen and/or radicals which are highly cytotoxic to bacteria $[4,5]$. The use of LAAAs to treat or prevent infectious diseases is known as photodynamic therapy (PDT) [6].

LAAAs are usually inorganic or organic dyes such as methylene blue (MB). LAAAs encapsulated in a polymer have shown to exhibit antimicrobial activity against a wide range of pathogenic bacteria [7-9]. The process used to encapsulate LAAAs into elastomers for medical application consisted of dipping the polymer into a LAAA solution con- taining a solvent capable of swelling the matrix; during this phase, the solution penetrates into the elastomer carrying the LAAA.

Hydrogels are water swellable materials having threedimensional polymeric network possessing both the mechanical properties of solids and diffusive transport of liquids. They have microscopic pores and elasticity, which provide their water holding and swelling capacity. These properties made hydrogels promising materials for biomedical applications [10]. Recently, polysaccharide-based hydrogels have got attraction in drug delivery system and dressing materials due to their extraordinary swelling properties, biodegradability, biocompatibility, and nontoxicity [11].

In our recent studies, we reported a class of polyacrylamide hydrogels [12] obtained by cross-linking of star-like branched copolymers of PAA with polysaccharide. It was shown that dextran-graft-polyacrylamide (D20-PAA) hydrogels exhibited the advantages in comparison with hydrogels based on linear polyacrylamide. D20-PAA hydrogels can be designed to have optimal water or biological fluid content in aqueous medium, good mechanical properties, shape stability, and softness similar to that of the soft surrounding tissue. Considering the facts of using of hydrogels as wound dressing, in the present work, we attempt to create the hydrogel/MB composites and study the antimicrobial 

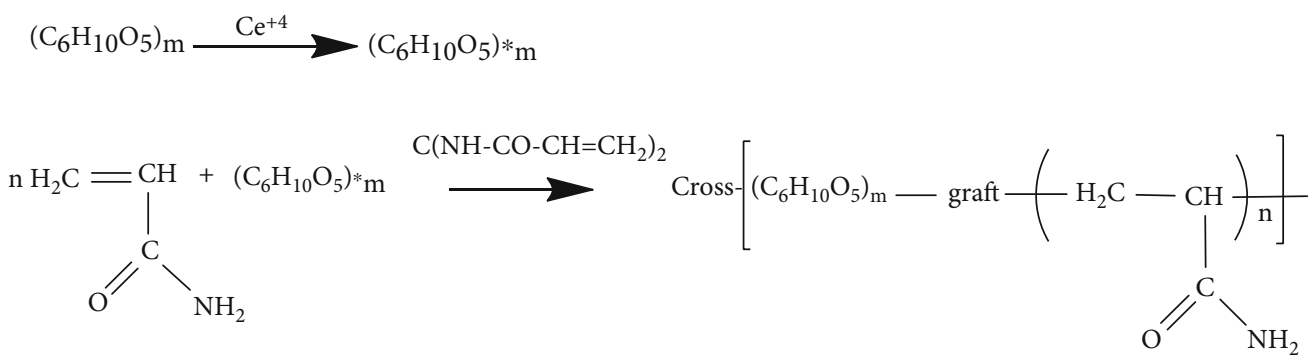

FIGURE 1: Schematic representation of hydrogel synthesis.

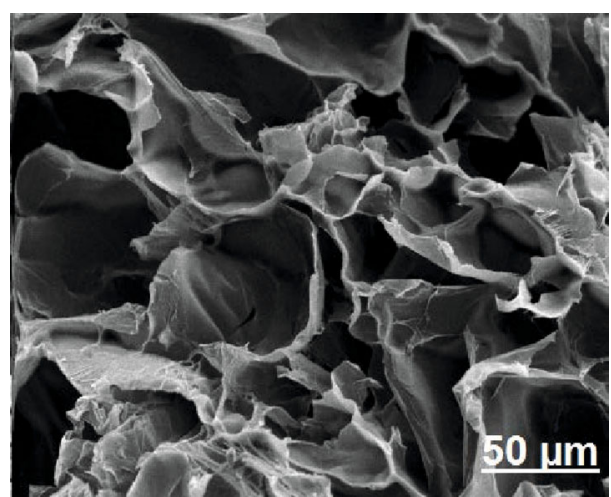

D20-PAA-x<smiles>CN(C)c1ccc2nc3ccc(=[N+](C)C)cc-3sc2c1</smiles>

Methylene blue

FIGURE 2: Internal structure of cross-linked copolymer networks and chemical structure of dye.

photodynamic effect. The monochromatic visible light at $\lambda=660 \mathrm{~nm}$ was used due to its minimal negative effects on human tissues and ability to stimulate regeneration processes [13].

\section{Materials and Methods}

2.1. Hydrogel Synthesis. Dextran-graft-polyacrylamide (D20PAA-x) hydrogels (Figure 1) with different concentrations of cross-linking agent $(x=0.2 ; 0.4$ and $0.6 \mathrm{wt}$. \%) were used. The synthesis of hydrogels is described in our previously work [12]. As precursors, we use acrylamide (AA) by SigmaAldrich. Dextran (D20) with $M_{\mathrm{w}}=20000 \mathrm{~g} / \mathrm{mol}$, cerium (IV) ammonium nitrate (CAN), N,N' -methylene-bis-acrylamide (MBA), and methylene blue $(\mathrm{MB})$ were purchased from Fluka. All supplied reagents were of analytical grade, and they were used without further purification. All procedures with hydrogels were performed in deionized water.

D20-g-PAA-x hydrogels were prepared by free radical polymerization using CAN as an initiator in the presence of cross-linking agent MBA [14] (Figure 1). The calculated amount of dextran $(0.02 \mathrm{mM})$ was dissolved in $25 \mathrm{~mL}$ of distilled water at $25^{\circ} \mathrm{C}$. This solution was purged by argon bubbling for $20 \mathrm{~min}$, and then, initiator CAN (0.03 mmol) was added. In $2 \mathrm{~min}$, AA $(0.05 \mathrm{~mol})$ and $\mathrm{MBA}(0.28,0.4$, or $0.6 \mathrm{~g}$ per $100 \mathrm{~g}$ of monomer AA) were poured into the reaction mixture. The formed hydrogel samples were taken out from the flask in $24 \mathrm{~h}$, washed with distilled water to remove unreacted monomer. Finally, the gels were dried at ambient temperature.
2.2. Determination of the Residual Acrylamide. To control the amount of residual AA in hydrogels, high-performance liquid chromatography (HPLC) was used. Water extracts were analyzed by the Waters Alliance HPLC System with a diode-array detector. The column Nucleosil C18 $(4 \times 250 \mathrm{~mm})$ was set at the temperature of $20^{\circ} \mathrm{C}$. The detection was conducted at the wavelength of $210 \mathrm{~nm}$. The mixture of methanol/redistilled water in the ratio $90 / 10 v / v$ was used as a mobile phase, at the flow rate of $1 \mathrm{~mL} \mathrm{~min}^{-1}$. Calibration curves for determining the contents of AA were constructed by preparing a series of solutions with known concentrations.

2.3. Preparation of Hydrogel Loaded with Methylene Blue. Loading of methylene blue into hydrogels was carried out by saturation of swollen hydrogels from aqueous solutions of methylene blue. The dye concentrations were 0.0001 and $0.001 \mathrm{wt}$. \%. Samples of $1 \times 1 \times 1 \mathrm{~cm}$ cubes $(\sim 1 \mathrm{~g})$ of cured hydrogels were immersed in a solution of $\mathrm{MB}$ for $12 \mathrm{~h}$. Internal structure of cross-linked copolymer networks and chemical structure of dye are shown in Figure 2.

2.4. UV-Vis Spectroscopy. The absorbance is measured using a Lambda 35 UV-Vis spectrophotometer (PerkinElmer, CA) in the absorbance mode (range $200-1000 \mathrm{~nm}$ ), at the absorption maximum of the methylene blue $\left(\lambda_{\max }=668 \mathrm{~nm}\right.$ [15]). In this case, the greater the absorbance, the higher the dye concentration. The concentration of dye in solution did not exceed $0.25 \times 10^{-3}$ wt. $\%$, to prevent dimerization of the dye and the appearance of an additional maximum of absorption. 
TABLE 1: Diffusion rate of dye and equilibrium dye concentration at sorption of methylene blue by hydrogel.

\begin{tabular}{lcccc}
\hline Sample & $V_{1}{ }^{*}\left(\mathrm{~min}^{-1}\right)$ & $V_{2}\left(\mathrm{~min}^{-1}\right)$ & $C_{\text {eq1 }} \times 10^{4}$ (wt. \%) & $C_{\text {eq2 }} \times 10^{4}$ (wt. \%) \\
\hline D20-PAA-0.2 & 0.10 & 0.40 & 4.16 & 1.46 \\
D20-PAA-0.4 & 0.08 & 0.35 & 3.64 & 1.59 \\
D20-PAA-0.6 & 0.07 & 0.33 & 2.94 & 1.61 \\
\hline
\end{tabular}

${ }^{*} V_{1}$ and $V_{2}$ : diffusion rate of $\mathrm{MB}$ into hydrogel and out of hydrogel; $C_{\mathrm{eq} 1}$ : equilibrium $\mathrm{MB}$ concentration in hydrogel at sorption; $C_{\mathrm{eq} 2}$ : equilibrium $\mathrm{MB}$ concentration in solution at sorption.

All optical spectra were acquired using quartz cuvettes with a $1 \mathrm{~cm}$ path length.

2.5. Dynamic Study of Dye Sorption and Desorption. The dynamic study of dye diffusion into hydrogel (sorption) was carried out by estimating the change in the absorption maximum of $\mathrm{MB}$ solutions.

For this aim, the hydrogel sample of $7 \mathrm{~mm}$ in diameter was put into of aqueous solution of methylene blue $\left(C=0.25 \times 10^{-3}\right.$ wt. \%). The mass ratio of hydrogels and solution in all experiments was $1: 4$. In $10 \mathrm{~min}$, the absorbance of the solution of nonabsorbed dye was measured. Further measurements were carried out at time intervals of $10 \mathrm{~min}$ for $150 \mathrm{~min}$ until the equilibrium concentration of the dye in solution was reached. The concentration of the nonabsorbed dye was determined by the calibration curve, and the amount of dye absorbed by the hydrogel was concluded (Table 1).

The diffusion of methylene blue out of the saturated hydrogels was similarly studied. In this case, the hydrogel samples loaded with MB were put into distilled water. The mass ratio of loaded with $\mathrm{MB}$ hydrogels and water in all experiments was $1: 4$. The initial concentration of dye in all hydrogels was $0.25 \times 10^{-3}$ wt. \%. In $10 \mathrm{~min}$, the optical density of the solution of desorbed dye was measured. Further measurements were carried out at time intervals of $10 \mathrm{~min}$ for $150 \mathrm{~min}$ until the equilibrium concentration of the dye in solution was reached. The concentration of the desorbed dye was determined by the calibration curve.

To analyze the adsorption and desorption rate $(\mathrm{V})$ of the dye, the first derivative of the dye concentration in solution vers time was calculated. For this purpose, the experimental dependence was linearized in logarithmic coordinates: time $(\ln t)$ vers absorbance $(\ln D)$ by fitting according to linear law; the slope of dependence obtained corresponds to the rate of dye diffusion. Slight deviations from the linear range do not exceed the permissible error of the experiment.

2.6. Antibacterial Studies. The LIKA-Led (Photonics Plus, Cherkasy, Ukraine) apparatus with laser emitters with wavelengths $\left(\lambda_{\text {ex }}\right)$ of $660 \mathrm{~nm}$ was used. An irradiation with light from a $100 \mathrm{~mW}$ laser for up to 20,30 , and 40 min results in an energy density of $21 \mathrm{~J} / \mathrm{cm}^{2}, 31.5 \mathrm{~J} / \mathrm{cm}^{2}$, and $42.1 \mathrm{~J} / \mathrm{cm}^{2}$, respectively.

The antimicrobial activity of methylene blue activated by red light was studied in a suspension of $S$. aureus $\left(10^{5} \mathrm{CFU} / \mathrm{mL}\right)$. A suspension of S. aureus $\left(10^{-5} \mathrm{CFU} / \mathrm{mL}\right)$ was prepared in a nonagar Müller-Hinton medium. $3.8 \mathrm{~mL}$ aliquots of the suspension were placed in tubes; then, $0.2 \mathrm{~mL}$ methylene blue solution of $0.02 \%$ wt. \% was added into suspension and incubated at $37^{\circ} \mathrm{C}$ throughout the experiment. Both suspension of $S$. aureus and suspension of $S$. aureus with methylene blue were irradiated with light $(660 \mathrm{~nm})$ from a $100 \mathrm{~mW}$ laser for up to $3 \mathrm{~min}$, resulting in an energy density of $0-18 \mathrm{~J} / \mathrm{cm}^{3}$. Each test was compared to the control because every $1-3 \mathrm{~h}$, there was a multiplication of bacteria and a doubling of their number. The bactericidal effect was evaluated in \% of CFU deaths relative to the control sample.

The antimicrobial activity of hydrogels loaded with methylene blue $(0.0005 \mathrm{wt}$. \%) in a suspension of $S$. aureus $\left(10^{5} \mathrm{CFU} / \mathrm{mL}\right)$ was studied. The hydrogel samples were placed into bacteria suspension in a mass ratio of hydrogel: suspension $=1: 4$. After $120 \mathrm{~min}$, the equilibrium concentration of $\mathrm{MB}$ in the solution was equal to $0.0001 \pm 0.00002 \%$ wt. \%. Further irradiation with light $(660 \mathrm{~nm}, 100 \mathrm{~mW})$ with interval of $20 \mathrm{~min}$ and an energy density of $6 \mathrm{~J} / \mathrm{cm}^{3}$ was carried out. The bactericidal effect was evaluated in \% of CFU deaths relative to the control sample.

A disk diffusion method was applied to study the antibacterial activity of the hydrogels. Wild strains of Staphylococcus aureus were used as Gram-positive bacteria models in the test. Wild strains of bacteria were obtained on an electric medium "Yolk-salt agar" [16]. The sensitivity of the selected strains to the action of light and methylene blue was carried out on solid medium. A suspension of the bacteria (of approximately $10^{5} \mathrm{CFU} / \mathrm{mL}$ ) was prepared to a particular standard, then spread evenly onto Müller-Hinton agar in a Petri dish.

The Petri dish was divided into four sectors hereinafter referred to as 1, 2, 3, and 4: for control (1), for irradiation with light (2), for hydrogel saturated with methylene blue (3), and for hydrogel saturated with methylene blue and irradiated with light (4). The hydrogel samples and their composites with $\mathrm{MB}$ were cut to $5 \mathrm{~mm}$ side squares and placed on Petri dishes with agar. Then, sector (2) and sector (4) were irradiated with light $\left(\lambda_{\text {ex }}=660 \mathrm{~nm}\right)$. The agar plates were placed in an incubating oven at $37^{\circ} \mathrm{C}$ and left for $24 \mathrm{~h}$.

The antimicrobial activity of MB-loaded hydrogels was assessed by analyzing the diameter of growth retardation [17]. Determination of colony-forming units (CFU) in bacterial suspensions was performed in the Goryaev chamber after staining an aliquot of the suspension with Acridine orange with a final dye concentration of $0.001 \mathrm{wt}$. \% [18]. CFU was determined using luminescence Acridine orange at a wavelength of $530 \mathrm{~nm}$. 


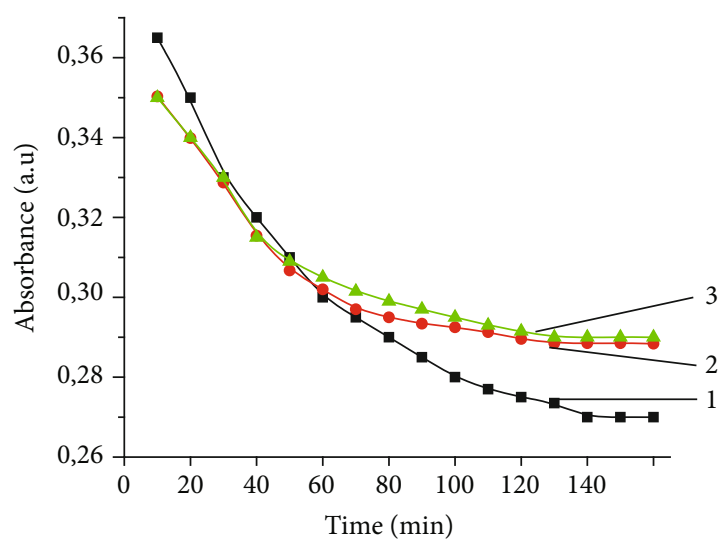

(a)

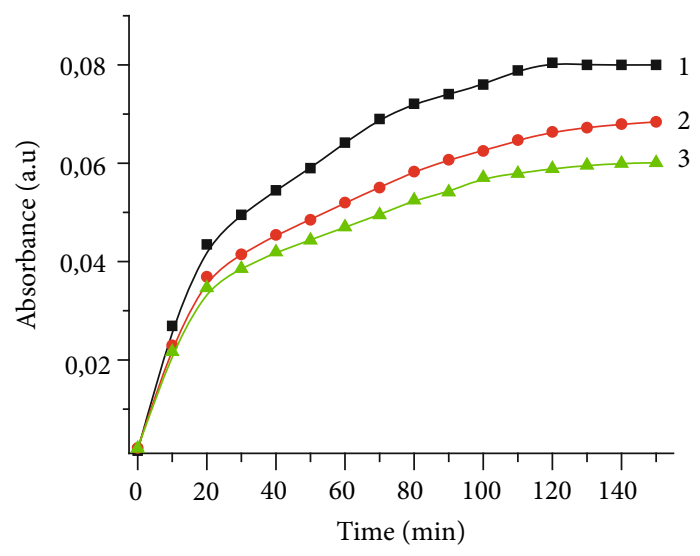

(b)

FIgURE 3: (a) The change in the absorption maximum of MB solution at $\lambda_{\max }=668 \mathrm{~nm}$ during dye diffusion in the hydrogel: 1 : D20-PAA-0.2, 2: D20-PAA-0.4, and 3: D20-PAA-0.6; (b) the change in the absorption maximum of the methylene blue solution during dye diffusion from the hydrogel: 1: D20-PAA-0.2, 2: D20-PAA-0.4, and 3: D20-PAA-0.6.

All experiments were performed in triplicate, and mean average values were reported.

\section{Results and Discussion}

3.1. Dynamic of Sorption/Desorption Studies. The diffusion rate of methylene blue into hydrogel samples was studied by changing the in the absorption maximum of $\mathrm{MB}$ solution into which the hydrogel was placed. The decrease in the absorbance of the solution indicates a decrease in the concentration of dye and, thus, an increase in its concentration in the hydrogel (Figure 3(a)).

Figure 3(b) represents the diffusion of methylene blue out of the saturated hydrogels into water. In this case, the increase in the absorbance of dye in the solution at the absorbance maximum corresponds to an increase in its concentration in solution. The equilibrium state of the dye desorption from the hydrogel was reached in $130-150 \mathrm{~min}$ for all samples.

The concentration of $\mathrm{MB}$ in the hydrogel in the equilibrium state is represented in Table 1. It is evident that the degree of cross-linking of the hydrogel decreases and the rate of diffusion of methylene blue into the hydrogel increases. It is caused by higher mesh size as it was demonstrated in [12]. The equilibrium concentration of the dye in the hydrogel is higher than in the solution, which indicates the intermolecular interactions of the dye molecules and the polymer matrix due to polar functional groups and ionic interactions in local volume of hydrogel. The lower cross-linking density of hydrogels leads to higher concentration of dye into polymer matrices after diffusion.

Figure 3(b) shows the strong dependence of the diffusion process on the concentration of cross-links in the hydrogel. The release rate of loaded MB out of hydrogel is the highest for the sample D20-PAA- 0.2 with the lowest cross-linking density, which corresponds to the largest mesh size in the network.

Thus, sorption/desorption properties of hydrogels are important factor for regulation of therapeutic concentrations

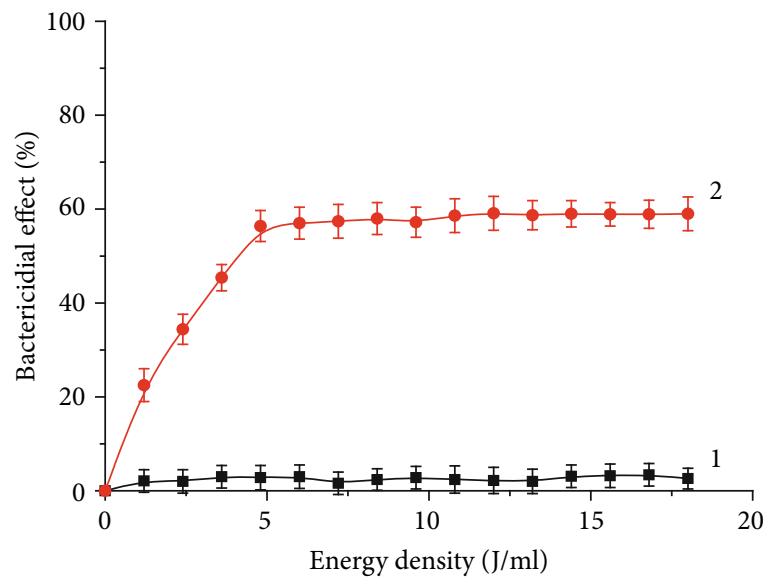

Figure 4: Bactericidal effect of red light $(660 \mathrm{~nm})(1)$ and methylene blue $\left(10^{-4}\right.$ wt. \%) activated by red light $(660 \mathrm{~nm})(2)$ in suspension of wild strains $S$. aureus $(\mathrm{M} \pm \mathrm{SD})$.

of the active substances in bacteria medium. It allows creating novel materials for biomedical application, wound dressing, for example. So, we use hydrogels D20-PAA-0.2 with the best diffusion properties for antibacterial studies.

\subsection{Antibacterial Studies}

3.2.1. Antibacterial Activity of Light-Activated $M B$ in the Bacterial Suspension. The study of antibacterial efficacy of red light $(660 \mathrm{~nm})$ against $S$. aureus in the bacterial suspension indicates no bactericidal action (Figure 4). This is due to the low energy of light quanta and the absence of photosensitizer targets in bacterial cells. It was also found that methylene blue at a concentration of $0.0001 \mathrm{wt}$. \% in the bacterial suspension does not show bactericidal properties relative to the bacterial strains. Then, red light irradiation and MB of 0.0001 wt. \% were combined and caused $20 \%$ reduction in CFU with a small radiation dose of about $2 \mathrm{~J} / \mathrm{cm}^{3}$. An increase of energy density to $6 \mathrm{~J} / \mathrm{cm}^{3}$ causes to inactivate $60 \%$ of CFU. Further increase in the radiation dose does 


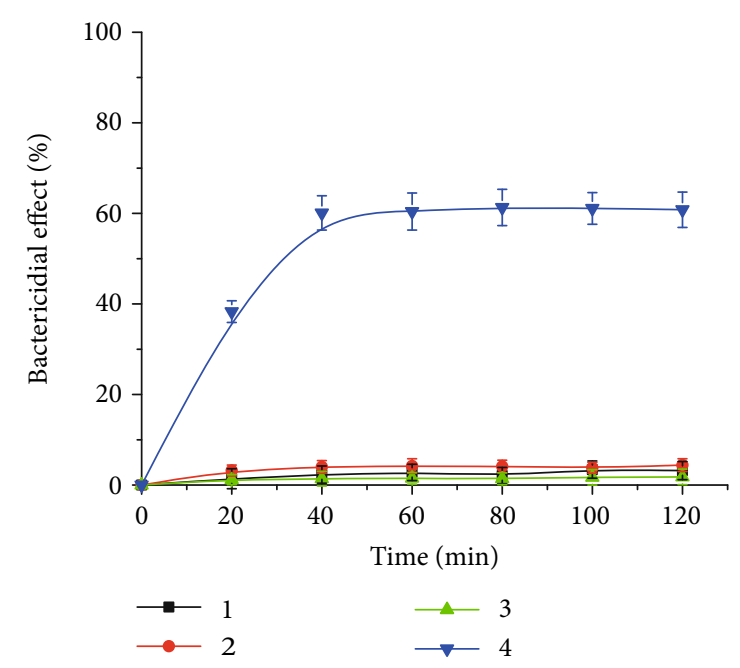

Figure 5: Bactericidal effect of red light $(660 \mathrm{~nm})(1)$, methylene blue (10 ${ }^{-4}$ wt.\%) (2), pure D20-PAA-0.2 (3), and D20-PAA-0.2 loaded with $\mathrm{MB}$ and activated by red light $(660 \mathrm{~nm})$ (4) in suspension of wild strains $S$. aureus. Energy density $=6 \mathrm{~J} / \mathrm{cm}^{3}$, $\mathrm{M} \pm \mathrm{SD}$.

not contribute to the growth of bactericidal activity. This may be due to the adaptation of $S$. aureus culture to the conditions created or the lack of oxygen or dye in solution.

\subsubsection{Antibacterial Activity of Hydrogel Loaded with $M B$ in} the Bacterial Suspension. The study of the diffusion of methylene blue out of the hydrogel into the solution has showed the gradual release of the dye into the environment in a short time. This allows for a long time to provide the required concentration of the active substance in the suspension of pathogenic bacteria. Considering obtained results, the bactericidal effect of hydrogel materials loaded with methylene blue in combination with visible light irradiation was investigated. D20-PA-0.2 of the lowest cross-linking density and the highest diffusion rate was separated as an MB container. First of all, it was shown that individual components such as pure hydrogel, light irradiation at $6 \mathrm{~J} / \mathrm{cm}^{3}$, and methylene blue do not possess antibacterial ability in bacterial suspension (Figure 5). At the same time, complex action of hydrogel composite D20-PAA-0.2/MB and light irradiation resulted in loss of $60 \%$ of the initial amount of CFU, with the incubation of the hydrogel in a solution of $40 \mathrm{~min}$.

\subsubsection{Antibacterial Activity of Hydrogels Loaded with $M B$ on} Solid Medium. The described above results of antibacterial studies in bacterial suspension indicate the antibacterial efficacy of composites based on cross-linked hydrogels loaded with methylene blue and activated by red light irradiation. Since such composites are promising materials for hydrogel dressings, it was interesting to study their antibacterial properties on the surface. Antibacterial efficacy is known to be caused by several factors such as the nature of the lightactivated antimicrobial agent, their diffusion rate and concentration, hydrogel nature, and bacteria strains.

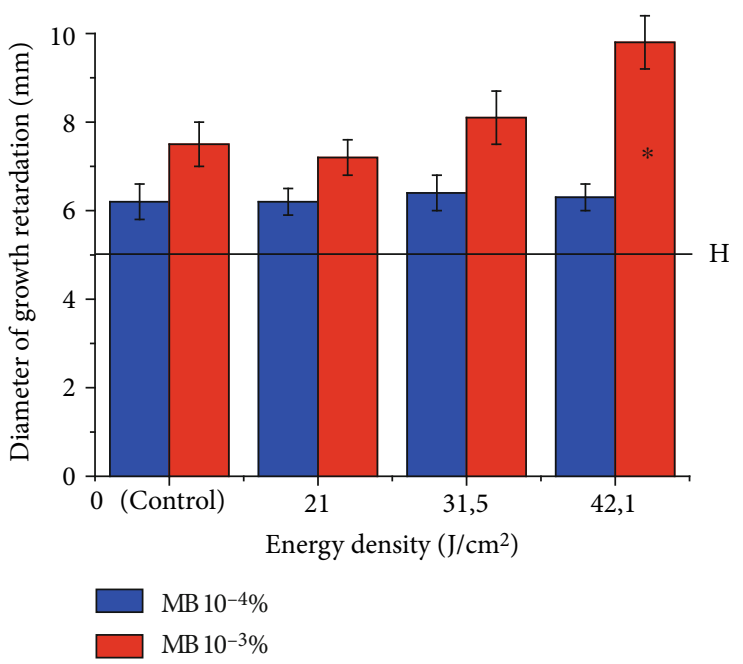

FIgURE 6: Bactericidal effect of D20-PAA-0.2 loaded with MB activated by red light $(660 \mathrm{~nm})$ on Müller-Hinton agar against wild strains $S$. aureus. $C_{\mathrm{MBA}}=10^{-3}, 10^{-4}$ wt. \%, H: diameter of the hydrogel sample, $\mathrm{M} \pm \mathrm{SD},{ }^{*} p<0.05$.

The bactericidal activity of hydrogels D20-PAA- 0.2 loaded with $\mathrm{MB}$ at low concentrations of $1 \times 10^{-3}$ and $1 \times$ $10^{-4}$ wt. \% against wild Staphylococcus aureus strains was investigated by the disc-diffusion method (Figure 6). As seen from Figure 6, irradiation of hydrogel loaded with $\mathrm{MB}$ at 1 $\times 10^{-4}$ wt. \% with different light energy density $(660 \mathrm{~nm})$ does not lead the appreciable bactericidal activity. When increasing the concentration of $\mathrm{MB}$ to $1 \times 10^{-3}$ wt. \%, its bactericidal activity increases and its dependence on radiation doses appears.

Thus, the hydrogel composites loaded with methylene blue as light-activated antimicrobial agent and irradiated with red light $(660 \mathrm{~nm})$ possess bactericidal effect both in suppressive and in solid media. It makes them promising materials for wound dressings and application for photodynamic therapy.

\section{Conclusions}

Hybrid cross-linked hydrogel dextran-graftedpolyacrylamide with different cross-linking density was synthesized as promising materials of a new generation for biomedical application. Methylene blue was used as the universal model of light-activated antimicrobial agent. Sorption/desorption process of dye into/out of hydrogels was studied. The release rate of absorbed MB into hydrogel was higher for the sample with the lowest cross-linking density. Antibacterial activity of hydrogel composites loaded with methylene blue as light-activated antimicrobial agent and irradiated with red light was tested in suspension and solid medium against wild strains $S$. aureus. The high antimicrobial efficacy was registered for both experiments. Thus, prepared hydrogel composites can be used as promising materials for wound dressings and photodynamic therapy. 


\section{Data Availability}

The [Dynamic of sorption/desorption studies] data used to support the findings of this study are included within the article. The [Antibacterial studies] data used to support the findings of this study are available from the corresponding author upon request.

\section{Conflicts of Interest}

The authors declare that there is no conflict of interest regarding the publication of this paper.

\section{References}

[1] W. Kim, W. Zhu, G. L. Hendricks et al., "A new class of synthetic retinoid antibiotics effective against bacterial persisters," Nature, vol. 556, no. 7699, pp. 103-107, 2018.

[2] P. S. Zolfaghari, S. Packer, M. Singer et al., "In vivo killing of Staphylococcus aureus using a light-activated antimicrobial agent," BMC Microbiology, vol. 9, no. 1, p. 27, 2009.

[3] M. Wilson, T. Burns, and J. Pratten, "Killing of Streptococcus sanguis in biofilms using a light-activated antimicrobial agent," Journal of Antimicrobial Chemotherapy, vol. 37, no. 2, pp. 377-381, 1996.

[4] K. Page, M. Wilson, and I. P. Parkin, "Antimicrobial surfaces and their potential in reducing the role of the inanimate environment in the incidence of hospital-acquired infections," Journal of Materials Chemistry, vol. 19, no. 23, pp. 38193831, 2009.

[5] V. Decraene, J. Pratten, and M. Wilson, "Cellulose acetate containing toluidine blue and rose bengal is an effective antimicrobial coating when exposed to white light," Applied and Environmental Microbiology, vol. 72, no. 6, pp. 4436-4439, 2006.

[6] R. A. Hsi, D. I. Rosenthal, and E. Glatstein, "Photodynamic therapy in the treatment of cancer," Drugs, vol. 57, no. 5, pp. 725-734, 1999.

[7] S. Perni, P. Prokopovich, C. Piccirillo, J. Pratten, I. P. Parkin, and M. Wilson, "Toluidine blue-containing polymers exhibit potent bactericidal activity when irradiated with red laser light," Journal of Materials Chemistry, vol. 19, no. 18, pp. 2715-2723, 2009.

[8] J. Gil-Tomás, S. Tubby, I. P. Parkin et al., "Lethal photosensitisation of Staphylococcus aureus using a toluidine blue Otiopronin-gold nanoparticle conjugate," Journal of Materials Chemistry, vol. 17, no. 35, pp. 3739-3746, 2007.

[9] K. Edwards, "New twist on an old favorite: gentian violet and methylene blue antibacterial foams," Advances in Wound Care, vol. 5, no. 1, pp. 11-18, 2016.

[10] Q. Chai, Y. Jiao, and X. Yu, "Hydrogels for biomedical applications: their characteristics and the mechanisms behind them," Gels, vol. 3, no. 1, p. 6, 2017.

[11] R. Parhi, "Cross-linked hydrogel for pharmaceutical applications: a review," Advanced Pharmaceutical Bulletin, vol. 7, no. 4, pp. 515-530, 2017.

[12] O. Nadtoka, N. Kutsevol, A. Naumenko, and P. Virych, "Photochemical synthesis and characterization of hydrogel-silver nanoparticle composites," Research on Chemical Intermediates, vol. 45, no. 8, pp. 4069-4080, 2019.
[13] Y. Wang, Y. Y. Huang, Y. Wang, P. Lyu, and M. R. Hamblin, "Red $(660 \mathrm{~nm})$ or near-infrared $(810 \mathrm{~nm})$ photobiomodulation stimulates, while blue $(415 \mathrm{~nm})$, green $(540 \mathrm{~nm})$ light inhibits proliferation in human adipose- derived stem cells," Scientific Reports, vol. 7, no. 1, article 7781, 2017.

[14] O. Nadtoka, N. Kutsevol, V. Krysa, and B. Krysa, "Hybrid polyacryamide hydrogels: synthesis, properties and prospects of application," Molecular Crystals and Liquid Crystals, vol. 672, no. 1, pp. 1-10, 2018.

[15] S. Perni, C. Piccirillo, A. Kafizas et al., "Antibacterial activity of light-activated silicone containing methylene blue and gold nanoparticles of different sizes," Journal of Cluster Science, vol. 21, no. 3, pp. 427-438, 2010.

[16] L. M. Carantonis and M. S. Spink, "A selective salt egg agar medium for pathogenic staphylococci," The Journal of Pathology, vol. 86, no. 1, pp. 217-220, 1963.

[17] M. Lehtopolku, P. Kotilainen, P. Puukka et al., "Inaccuracy of the disk diffusion method compared with the agar dilution method for susceptibility testing of Campylobacter spp," Journal of Clinical Microbiology, vol. 50, no. 1, pp. 52-56, 2012.

[18] C. Camacho-Fernández, D. Hervás, A. Rivas-Sendra, M. P. Marín, and J. M. Seguí-Simarro, "Comparison of six different methods to calculate cell densities," Plant Methods, vol. 14, no. 1, p. $30,2018$. 